\title{
Nano-sized glass as an economically viable and eco-benign support to anchor heteropolyacids for green and sustainable chemoselective oxidation of sulfides to sulfoxides
}

\author{
SOMAYEH ZOLFAGHARINIA, ESKANDAR KOLVARI*, NADIYA KOUKABI \\ and MALIHEH M HOSSEINI \\ Department of Chemistry, Semnan University, Semnan, Iran \\ E-mail: kolvari@semnan.ac.ir
}

MS received 11 December 2016; revised 17 April 2017; accepted 17 June 2017; published online 2 September 2017

\begin{abstract}
In this work, glass wastes were employed as cost-effective supports for the immobilization of phosphomolybdic acid (5-25 wt.\% PMA) through an impregnation method. The highly efficient and retrievable nanocatalyst named nano-glass waste-supported phosphomolybdic acid (n-GW/PMA) was fully characterized by several techniques such as: XRD, FE-SEM, EDX, FT-IR and TGA. The catalytic performance of the assynthesized heterogeneous nanocatalyst was effectively investigated for the chemoselective oxidation of sulfides to sulfoxides in the presence of $30 \% \mathrm{H}_{2} \mathrm{O}_{2}$ as an oxidant at room temperature under solvent-free condition. Optimization of the reaction conditions was performed by means of central composite design (CCD), which is one of the powerful response surface methodologies. Based on the results obtained under the optimum condition, the sample of $16 \mathrm{wt} . \%$ of PMA loading offered high conversion rates and yields (97\%). Besides, the beneficial points of the prepared catalyst were its recoverability and reusability for several reaction cycles, low-cost and toxicity, easy availability and facile production.
\end{abstract}

Keywords. Nano-glass; phosphomolybdic acid; impregnation method; nanocatalyst; sulfide oxidation; reusability.

\section{Introduction}

During the last decades, in the light of green chemistry approaches, implementation of more sustainable practices in industries has been undergoing considerable alteration. Green chemistry strives to protect the health and the environment in a cost-effective manner. In this context, significant improvement has been made for designing safer chemicals and eco-friendly solvents and catalysts. Catalysis is the central focus of green chemistry research in $21^{\text {st }}$ century due to its applications in various systems with reduced toxicity, renewable and benign energy, and high reaction efficiency. Based on these facts, preparation of heterogeneous analogues of the most commonly used soluble and homogeneous catalysts can be approached through their immobilization on different insoluble supports. In the light of this situation, the simplification of catalyst removal and the limitation in the amounts of wastes produced have been accomplished. However, in such heterogeneous systems, catalyst recovery through filtration or precipitation would be a major issue because of considerable timeand energy-consumption. Additionally, on account of the heterogeneous nature of the support materials in reaction media, steric and diffusion factors, the immobilized catalysts show a substantial decrease in the activity and selectivity. ${ }^{1,2}$ Besides, in the systems where a great proportion of active species are deep inside the supporting matrix, the reactants encountered difficulties for accessing to the catalytic active sites. ${ }^{3}$ Hence, for the economic viability preservation, the designed heterogeneous systems must exhibit the advantages with respect to minimization of waste formation, comparable or higher catalytic activities and selectivity over the existing homogenous routes. To address these challenges, nanoparticles (NPs), the alternative soluble matrixes,

\footnotetext{
*For correspondence
}

Electronic supplementary material: The online version of this article (doi:10.1007/s12039-017-1348-5) contains supplementary material, which is available to authorized users. 
have been applied to support homogeneous catalysts or reagents. The advantages of the support materials lie with increasing the surface area on the basis of their nanometer sizes, higher catalyst loading capacity and dispersion comparable to the most common support matrixes. ${ }^{4-6}$

Polyoxometallates (POMs) stand for a diverse family of anionic metal oxide molecular species, which span a rich variety of sizes and shapes, with outstanding properties and functions as acid and redox catalysts both in the homogeneous and heterogeneous media. ${ }^{7}$ Heteropolyacids (HPAs), an extremely large and varied class of polyoxometalates, as powerful oxidants, can display fast reversible multi-electron redox transformation under mild condition. ${ }^{8}$ Thanks to their unique properties, heteropolyacids can open up the opportunity for catalyzing the reactions in both solution and solid state lowering the production of wastes or byproducts. There is a plethora of large scale industrial transformations catalyzed by HPAs including alcohol dehydration, alkylation and esterification, etc. ${ }^{9,10}$ However, the main drawbacks of HPA are pertained to their low surface areas and high solubility in polar solvents which render difficulties in respect of their recovery and catalytic useful life. Addressing these issues, such catalytically efficient HPAs are frequently immobilized onto the insoluble, easily separable solid matrixes with high surface areas ${ }^{7,11-13}$ The attractive feature related to the supported HPAs is the fact that they are more active than typical solid acids. So far, numerous support materials such as silica, ${ }^{14}$ zeolite, ${ }^{15}$ alumina, ${ }^{16}$ active carbon ${ }^{17}$ and ion-exchange resin ${ }^{18}$ have been employed for the immobilization of HPAs. However, the alternative routes show some problems including the formation of deposit (coke) on the catalyst surface which makes these types of supported catalysts to be susceptible against deactivation during organic reaction ${ }^{7}$ In addition, HPAs supported on solid matrixes are prone to leaching in polar solvents which limit their reusability. Therefore, there is still much room for the improvement of such systems through the application and design of stable, inexpensive, active and available solid support for binding of HPAs. In this context, we postulated that the application of waste materials or products as solid supports for the immobilization of HPAs could provide a golden chance which one could achieve three goals, at the same time: (i) The waste materials or products can be recycled and in the subsequent step, the drastic impacts on the environment can be mitigated. (ii) An efficient solid heterogeneous catalyst can be prepared through the heterogenization of HPAs. (iii) An organic transformation can be rapidly catalyzed in a mild reaction condition.
Glass in many forms such as packaging or container glass (bottles and jars), flat glass (windows and windscreens), bulb glass (light globes) and cathode ray tube glass (TV screens, monitors, etc.), have a limited life. Therefore, it is essential to reuse or recycle them so as to prevent environmental problems related to their stockpiling or sending to the landfill. ${ }^{19}$ As a result, we thought that the utilization of nano-glass waste for supporting heteropolyacids could go a long way towards the simultaneous reaching the above-mentioned targets. In this context, recently we reported nano-glass waste-supported sulfonic acid as an accessible, stable, recoverable, non-toxic and inexpensive solid acid nanocatalyst for multicomponent reactions. ${ }^{20}$ However, to the best of our knowledge there are no literature reports on the exploitation of glass wastes for the immobilization of the highly applicable heteropolyacids. Among heteropolyacids, we chose phosphomolybdic acid (PMA) because it is one of the least expensive commercially available one. In continuation of our attempts for the preparation of different solid acid catalyst, ${ }^{21-28}$ the present study consists of the synthesis of a novel solid acid nanocatalyst named nano-glass waste-supported phosphomolybdic acid (n-GW/PMA) and its application for the facile, fast, highly efficient, and chemoselective oxidation of sulfides to sulfoxides in the presence of $\mathrm{H}_{2} \mathrm{O}_{2}$ as oxidant under solvent-free conditions. Afterwards, the newly synthesized n-GW/PMA was fully characterized through FT-IR, EDX, FE-SEM and TGA techniques. The specific advantages of the catalyst are related to its high stability, facile recoverability, reusability, easy accessibility and low cost.

\section{Experimental}

\subsection{Chemicals and apparatus}

All the chemicals were purchased from Merck and Aldrich companies and used without any further purification. Glass wastes were provided from Sandis (Iran). Powder making of the glass waste was carried out through a two-cup planetary ball mill. The products purity was checked through thin layer chromatography (TLC) on glass plates coated with silica gel 60 F254 using n-hexane/ethyl acetate mixture as mobile phase. All yields refer to the isolated products after purification. Products were characterized by melting points and spectroscopic data (FTIR, ${ }^{1} \mathrm{H}$ NMR and ${ }^{13} \mathrm{C}$ NMR spectra). Melting points were determined in open capillaries using an Electrothermal 9100 without further corrections. Fourier transform infrared spectroscopy (FT-IR) was recorded on a Shimadzu 8400 s spectrometer using $\mathrm{KBr}$ pressed powder discs. ${ }^{1} \mathrm{H}$ - and ${ }^{13} \mathrm{C}$-NMR spectra were recorded with a Bruker Avance 400 Ultrashield NMR spectrometer, in pure 
Table 1. Oxidation of sulfides to sulfoxides in the presence of n-GW/PMA ${ }^{\mathrm{a}}$.

\begin{tabular}{|c|c|c|c|c|c|}
\hline Entry & Sulfide & Sulfoxide & $\mathrm{T}[\mathrm{min}]$ & Yield [\%] & Selectivity (\%) \\
\hline 1 & & & 25 & 91 & 100 \\
\hline 2 & & & 15 & 96 & 100 \\
\hline 3 & & & 10 & 95 & 100 \\
\hline 4 & & & 10 & 95 & 100 \\
\hline 5 & & & 65 & 83 & 100 \\
\hline 6 & & & 10 & 97 & 100 \\
\hline 7 & & & 20 & 95 & 100 \\
\hline 8 & & & 25 & 88 & 100 \\
\hline 9 & & & 50 & 91 & 100 \\
\hline 10 & & & 60 & 92 & 100 \\
\hline
\end{tabular}

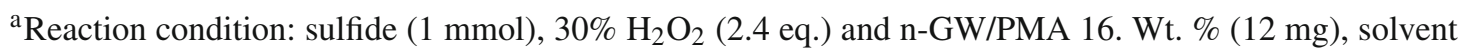
free, r.t.

${ }^{\text {b }}$ Yields refer to isolated products. 
deuterated chloroform with tetramethylsilane (TMS) as the internal standard. Thermogravimetric analyses (TGA) were performed on a DuPont 2000 thermal analysis apparatus at a heating rate of $5^{\circ} \mathrm{C} \mathrm{min}^{-1}$ under air atmosphere. X-ray diffraction (XRD) was recorded by Philips using $\mathrm{Cu}-\mathrm{K} \alpha$ radiation of wavelength $1.54 \AA$. Field emission scanning electron microscope (FE-SEM) images were obtained through Tescanvega II XMU Digital Scanning Microscope. The EDX spectra were acquired with the aid of a Mira 3-XMU scanning electron microscope equipped with an energy dispersive X-ray spectrometer operating. A central composite design (CCD) was performed to optimize the reaction condition for the oxidation method. 20 data analyses were carried out using Design-Expert trial version 7.0.0. (Stat-Ease Inc., Minneapolis).

\subsection{Preparation of the neat glass waste powder}

After washing glass wastes with $200 \mathrm{~mL}$ methanol and ethanol, they were turned into fine powder. Then, the prepared powder $(20 \mathrm{~g})$ was carefully washed with excess amount of distilled water three times. Afterwards, precipitated glass powder was filtered and rinsed with excess amount of water and then dried at $120^{\circ} \mathrm{C}$.

\subsection{Synthesis of the glass waste-supported phosphomolybdic acid}

The n-GW/PMA with the loadings of 5-25 wt.\% were prepared by the aid of the wetness impregnation method. Typically, $2 \mathrm{~g}$ of $\mathrm{n}-\mathrm{GW}$ was impregnated with an aqueous solution of PMA (0.06-0.375 g in 10-50 mL double distilled water). The mixture was vigorously stirred overnight at room temperature. Then, the water was completely evaporated using a rotary evaporator and the green sample was prepared after drying and calcination at $150^{\circ} \mathrm{C}$ for $2 \mathrm{~h}$.

\subsection{General procedure for the catalytic oxidation of sulfides}

To a reactor equipped with a stirrer bar, a mixture of sulfide ( $1 \mathrm{mmol}), 30 \% \mathrm{H}_{2} \mathrm{O}_{2}$ (2.4 eq.) and the $\mathrm{n}-\mathrm{GW} / \mathrm{PMA} 16 \mathrm{Wt} . \%$ $(12 \mathrm{mg})$ was added and allowed to be stirred at room temperature for appropriate time indicated in the Table 1. The progress of the reaction was monitored by TLC (n-hexane: EtOAc, 9: 1). After the reaction was completed, the mixture was diluted with $\mathrm{Et}_{2} \mathrm{O}(10 \mathrm{~mL})$ and the catalyst was separated through centrifugation. Then, the combined organic layers were dried over anhydrous $\mathrm{Na}_{2} \mathrm{SO}_{4}$ and diethyl ether was evaporated under reduced pressure to afford the pure products in $83-97 \%$ yields.

2.4a 4, 4'-sulfinyldiphenol (Table 1, entry 6): IR (KBr) $v / \mathrm{cm}^{-1}$ : 3236, 2923, 1583, 1496, 1438, 1365, 1139, 1000, 721, 690; ${ }^{1} \mathrm{H}-\mathrm{NMR}$ (DMSO-d 6 , $\left.400 \mathrm{MHz}\right) \delta_{H}(\mathrm{ppm})$ : $10.08(\mathrm{~s}, 2 \mathrm{H}), 6.8-7.7(\mathrm{dd}, 8 \mathrm{H}) ;{ }^{13} \mathrm{C}$ NMR (DMSO-d 6 , $400 \mathrm{MHz}): \delta_{C}(\mathrm{ppm}): 116.52,127.04,135.41,160.07$.

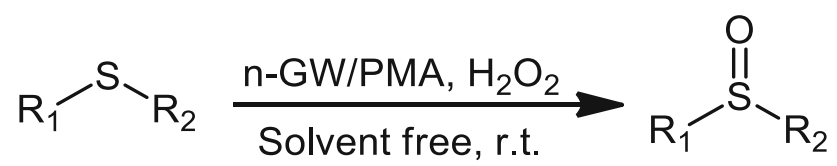

Scheme 1. Nano-GW/PMA catalyzed oxidation of sulfides to sulfoxides using $\mathrm{H}_{2} \mathrm{O}_{2}$.

$2.4 \mathrm{~b}$ dibenzo[b,d]thiophene 5-oxide (Table 1, entry 10): White solid, M.p.: $215-217^{\circ} \mathrm{C}$. IR (KBr) $v / \mathrm{cm}^{-1}$ : 3083, 2982, 1591, 1452, 1288, 1164, 1047, 711, 698; ${ }^{1}$ H-NMR $\left(\mathrm{CDCl}_{3}, 400 \mathrm{MHz}\right) \delta_{H}(\mathrm{ppm}): 7.5-7.8(\mathrm{~m}, 8 \mathrm{H})$. ${ }^{13} \mathrm{C} \mathrm{NMR}\left(\mathrm{CDCl}_{3}, 400 \mathrm{MHz}\right): \delta_{C}(\mathrm{ppm}): 120.57,121.132$, $129.35,130.57,132.87,136.65$.

\subsection{Investigation of the catalytic activity of $n$-GW/PMA for the oxidation of sulfides to sulfoxides}

Owing to the fact that organic sulfoxides have been widely used in variable pharmaceutical and agrochemical sectors, the selective oxidation of sulfides to sulfoxides are considered as an important transformation in organic synthesis. ${ }^{29,30}$ As a consequence, we were inspired by the investigation of the catalytic activities of our synthesized n-GW/PMA as a heterogeneous nanocatalyst for the chemoselective oxidation of structurally diverse sulfides to sulfoxides in the presence of $30 \% \mathrm{H}_{2} \mathrm{O}_{2}$ as an oxidant (Scheme 1).

Aiming at establishing the optimum reaction condition, we studied the model reaction of 4, 4'-thiodiphenol oxidation. At first, we tested the model reaction without any catalyst but the results were unsatisfactory (i.e., the yields were negligible (28\%) in the prolonged reaction time (2 h)). Therefore, it was understood that the presence of the catalyst in the reaction was essential. Then, for acquiring the best condition for the oxidation reaction, central composite design (CCD) was chosen. When the number of effective parameters are lower than/or equal to four, central composite design (CCD), as one of the most applicable types of response surface model (RSM), could be helpful.

At the outset, preliminary experiments were performed for the evaluation of the suitable parameters and for the determination of the experimental domain. Considering these experiments, the effects of catalyst amount (A), PMA loading (B) and $\mathrm{H}_{2} \mathrm{O}_{2}$ concentration (C), were studied on reaction yield as response in $10 \mathrm{~min}$. Optimization is performed in condition at which the optimal parameters can be employed for all of the used derivatives. Since time was variable about each derivatives, it was not optimized. Therefore, in the optimization process, each run was tested in $10 \mathrm{~min}$. A five-level CCD of three independent variables with their corresponding values was displayed in Table S1 in Supporting Information.

Based on the CCD method, total number of experiments was found to be twenty, which were comprised of eight full factorial points, six axial points and six central points. Conditions of 20 trials in addition to their respective yields are shown in Table S2 (See Supporting Information). 
With the aid of performing analysis of variance (ANOVA) on the model, valuable information about significance of fitted model and its terms were achieved. According to the Table S3 (Supporting Information), the model $p$-value and lack of fit were lower and higher than 0.05 , respectively indicating the fact that, fitted model was significant in confidence level of $95 \%$ and it was not needed to reduce to lower orders. Besides, the amounts of $R$-squared and adj $R$-squared were above 0.9 and close to each other which was a testimony for the high accuracy and reliability of the fitted model in prediction of the reaction yield. It was meant that there was a good agreement between experimental and the predicted responses which approved the suitability of the following fitted model regarding the significantly linear, quadratic and interaction terms based on their $p$-values.

$$
\begin{aligned}
\mathrm{Y}= & +1.90313+6.42339 \mathrm{~A}+4.54018 \mathrm{~B}+16.29883 \mathrm{C} \\
& +0.030000 \mathrm{AB}--0.16667 \mathrm{AC}-0.50000 \mathrm{BC} \\
& -0.27761 \mathrm{~A}^{2}-0.11497 \mathrm{~B}^{2}-1.07532 \mathrm{C}^{2}
\end{aligned}
$$

It can be found from the response equation that the three A, B and $\mathrm{C}$ parameters showed a linear effect on the product yield, therefore all factors (i.e., the amount of catalyst, PMA loading and $\mathrm{H}_{2} \mathrm{O}_{2}$ concentration) were important in progressing of the reaction. In order to investigate the main interaction effects between two parameters on the yield of reaction, three and two dimensional profile of yield versus a pair of parameters were used (Figure S18 in Supporting Information).

Based on their $p$-values, it could be realized that the interaction of $\mathrm{AB}, \mathrm{AC}$ and $\mathrm{BC}$ were significant; therefore, the simultaneous changing in the catalyst amount, PMA loading and $\mathrm{H}_{2} \mathrm{O}_{2}$ concentration could alter the product yield. When catalyst amount (12 mg), PMA loading (16 wt.\%) and $\mathrm{H}_{2} \mathrm{O}_{2}$ (2.4 equiv.) were applied in the reaction, the product yields and the desirability were $96.92 \%$ and 0.99 , respectively. The main target of this design was to optimize and maximize the yield of the reaction corresponded to the conditions of an experiment in which the response equation was maximized. In this work, determination of the optimal conditions was done with the help of desirability function by using DesignExpert 7.0.0. It was initially selected desired goals for each factor and for response to obtain the maximum product yield with high desirability function (close to one). Then, conditions possessing high desirability were tested three times. Negligible difference between the average yields and the prediction values of software, confirms the high accuracy and precision of optimum conditions. The results showed that $12 \mathrm{mg}$ of the catalyst, 16 wt. $\%$ of PMA loading and 2.4 equivalent of
$\mathrm{H}_{2} \mathrm{O}_{2}$ were the optimum conditions for the synthesis of 4, 4'-sulfinyldiphenol (Figure S19).

In order to study the effects of solvents on the reaction efficiency, the model reaction was carried out under optimum condition in the presence of $\mathrm{EtOH}, \mathrm{MeOH}, \mathrm{CH}_{3} \mathrm{CN}, \mathrm{H}_{2} \mathrm{O}$, and $\mathrm{CH}_{2} \mathrm{Cl}_{2}$. However, the best results in terms of reaction yields and time were related to the reactions operated under solvent free conditions.

The obtained results encouraged us to extent the application of this optimized catalytic protocol to the oxidation of various sulfides (Table 1). As could be observed from the table 1, a variety of sulfides like dialkyl sulfides, alkyl aryl sulfides and other sulfides with different functional groups could yield the corresponding sulfoxides with high conversion rate and outstanding selectivity. Additionally, under the designed reaction condition in the presence of the novel n-GW/PMA catalyst, the oxidation reactions were carried out smoothly without detecting any over oxidation products such as sulfones. Moreover, the steric effects of some sulfide derivatives were clear in this catalytic system. For example, the oxidation of diphenyl sulfide in comparison with isopropyl sulfide was performed in much longer reaction time which afforded a much lower reaction yields (Table 1, entries 4 and 5). The structures of some of the products were well characterized by using ${ }^{1} \mathrm{H}-\mathrm{NMR}$ and ${ }^{13} \mathrm{C}-\mathrm{NMR}$ spectral data.

\section{Results and Discussion}

The novel nano-glass waste supported phosphomolybdic acid (n-GW/PMA) was synthesized through impregnation method as briefly showed in Scheme 2. In order to synthesize the desired novel catalyst, firstly, nano-glass waste was prepared by crushing and powder making of the glass waste provided. Afterwards, an impregnation reaction occurred between the n-glass waste and PMA. At the subsequent step, the obtained catalyst was dried and calcinated at $150^{\circ} \mathrm{C}$ for $2 \mathrm{~h}$ and allowed to cool to room temperature. In order to explore the best amounts of PMA loaded on the n-GW support, a series of catalysts containing 5-25 wt.\% PMA was synthesized. The results obtained showed that the catalyst with $16 \mathrm{wt} \%$ loading exhibited better performance compared to the other catalysts. The newly prepared catalyst was characterized through FT-IR, FE-SEM, XRD, EDX and TGA techniques.

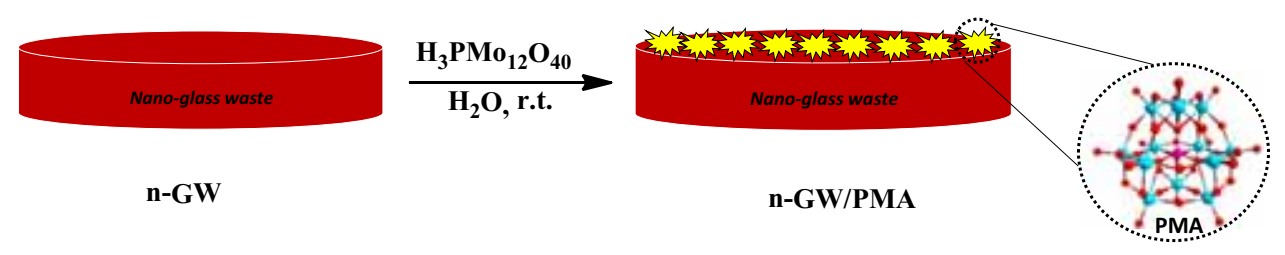

Scheme 2. Preparation of nano-glass waste-supported phosphomolybdic acid. 


\subsection{Characterization of $n-G W / P M A$}

3.1a FT-IR spectra of the samples: FT-IR spectra were employed to establish the presence of PMA on the n-glass waste support. Figure 1 showed the FTIR spectra of the n-GW, n-GW/PMA (10, 16 and 25 wt.\%) and PMA. Curve (a) in Figure 1 shows the infrared spectrum of n-GW. About Figure 1a, the bands appeared at 3523 and $1623 \mathrm{~cm}^{-1}$ are respectively assigned to the stretching and bending modes of the $\mathrm{SiOH}$ groups and the adsorbed water. The strong absorbance at 1066, 775 and $468 \mathrm{~cm}^{-1}$ are related to the asymmetric stretching, symmetric stretching and bending modes of $\mathrm{SiO}_{2}$, respectively ${ }^{31}$ The FT-IR spectrum of n-GW/PMA displayed the absorbance at 1058, 962,882 and $792 \mathrm{~cm}^{-1}$ which are attributed to the the symmetric stretching of the central-atom-oxygen $(\mathrm{P}-\mathrm{O})$ bond of the $\mathrm{PO}_{4}$ tetrahedron, the asymmetric stretching of the peripheral-atom-terminal-oxygen (Mo-O) bond, and the Mo-O-Mo stretching of the inter- and intraoctahedral bridges of the trimetallic group, respectively (Figure 1c). ${ }^{32}$ These observations confirmed the fact that the existence of PMA on support was in the Keggin structure.The vibrations due to molecular water present in the n-glass waste support and phosphomolybdic acid are identified at about 1627 and $3560 \mathrm{~cm}^{-1}$. The FTIR spectrum of PMA (Figure 1e) reveals that the bands in n-GW/PMA are corresponded to those for the PMA (i.e., the peaks at $1064,960,880$ and $779 \mathrm{~cm}^{-1}$ ). The FT-IR spectra of the different loading of PMA into nGW indicated that most of characteristic bands of the parent Keggin structure could be found in PMA fingerprint region (1250-500 $\mathrm{cm}^{-1}$ ). These findings clearly confirmed that the phosphomolybdic acid molecules are covalently bound to the n-GW support.

3.1b X-ray diffraction analysis: Figure 2 shows the $X R D$ patterns of $n-G W$, pure PMA and 16 wt.\% PMA supported on n-GW. According to Figure $2 \mathrm{a}, \mathrm{n}-\mathrm{GW}$ possessed the amorphous structure with the broad peak around $2 \theta$ equal to $27^{\circ} .^{33}$ As can be seen from Figure $2 c$, the crystalline peaks appeared between $2 \theta$ equal to $10-45^{\circ}$ are attributed to the purely crystalline form of PMA. ${ }^{34}$ The XRD pattern of n-GW/PMA $16 \%$ shows some reflections of Keggin structure PMA at 20-35 $(2 \theta)$ (Figure 2b). From the XRD analysis, it can be concluded that the Keggin structure of the $\mathrm{H}_{3} \mathrm{PMo}_{12} \mathrm{O}_{40}$ was maintained after supporting on $\mathrm{n}-\mathrm{GW}$.

3.1c Thermogravimetric (TGA) analysis: Thermogravimetric analysis (TGA) was used for the evaluation of materials in terms of their thermal stability, chemisorption and bond formation. Figure 3 shows

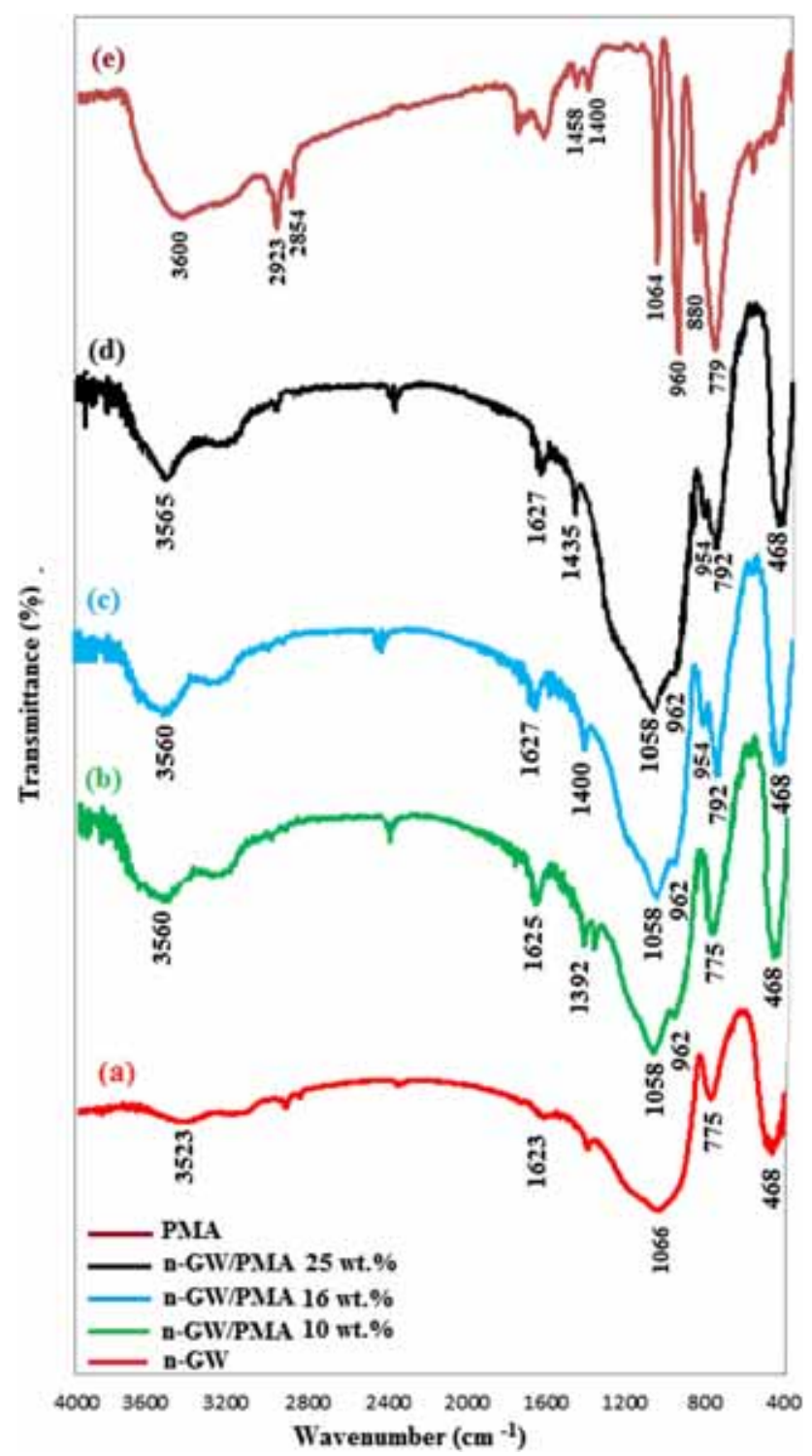

Figure 1. FT-IR spectra of (a) n-GW, (b-d) n-GW/PMA $10-25$ wt. $\%$ and (e) PMA.

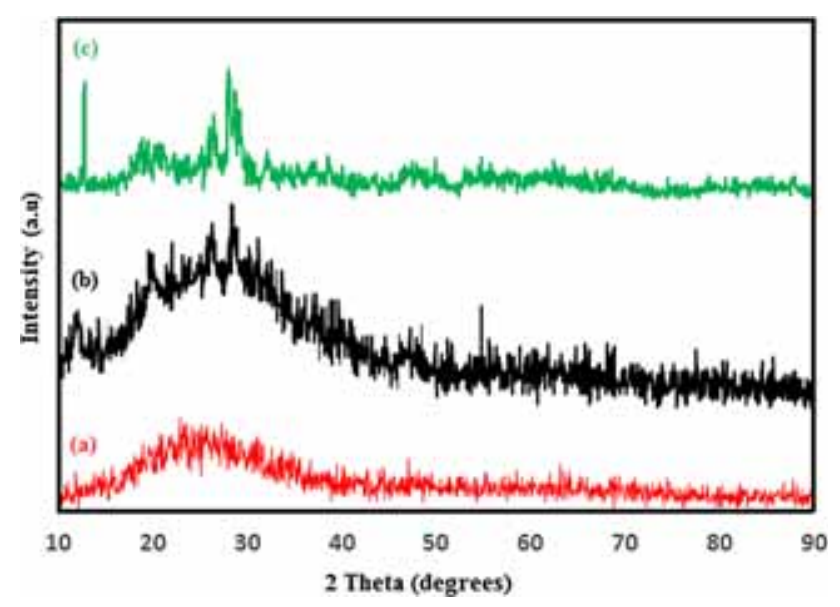

Figure 2. XRD spectra of (a) n-GW, (b) n-GW/PMA 16 wt.\% and (c) bulk PMA. 


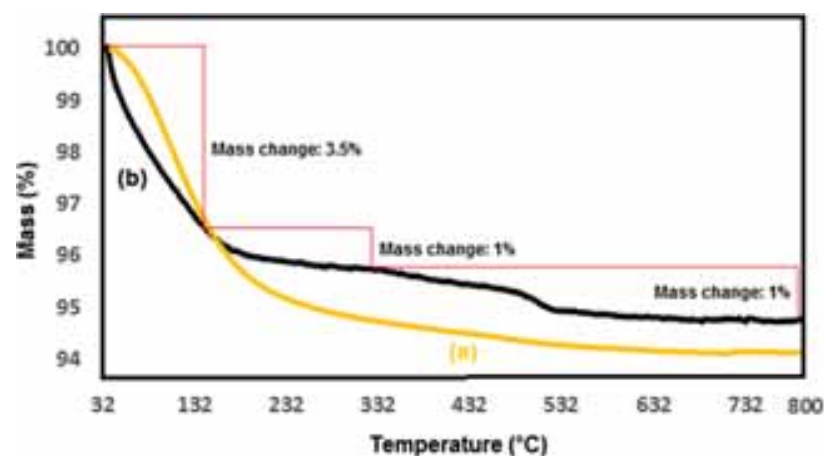

Figure 3. TGA curves of (a) n-GW and (b) n-GW/PMA 16 wt.\%.

the TGA results for the $n-G W$ and n-GW/PMA 16 wt.\% in the temperature ranging from 30 to $800^{\circ} \mathrm{C}$. As can be observed from Figure $3 \mathrm{a}$, the naked n-GW support shows a mass loss of about 4 wt.\% in the temperature up to $100^{\circ} \mathrm{C}$ relating to the loss of physically adsorbed water. The steadily dehydroxilaytion of $\mathrm{n}-\mathrm{GW}$ was occurred at the temperature lower than $600^{\circ} \mathrm{C}$ which caused 2 wt.\% loss. ${ }^{35}$ According to Figure $3 \mathrm{~b}$, nGW/PMA exhibited the weight loss of 3.5 wt. $\%$ up to $100^{\circ} \mathrm{C}$, attributed to removal of the physisorbed water. The second loss of weight (about 1 wt.\%) between $130-300^{\circ} \mathrm{C}$ was ascribed to the dehydroxylation of chemisorbed water and the strongly adsorbed water on the $\mathrm{n}-\mathrm{GW}$ support. ${ }^{36}$ The continuous weight loss (1 wt.\%) observed with further heating up to $800^{\circ} \mathrm{C}$ was due to the loss of water of the supported heteropolyacid and decomposition of the keggin units to give $\mathrm{MoO}_{3} \cdot{ }^{37,38}$ The results showed that the thermal stability of $n-G W / P M A$ was more than $300^{\circ} \mathrm{C}$ which could be
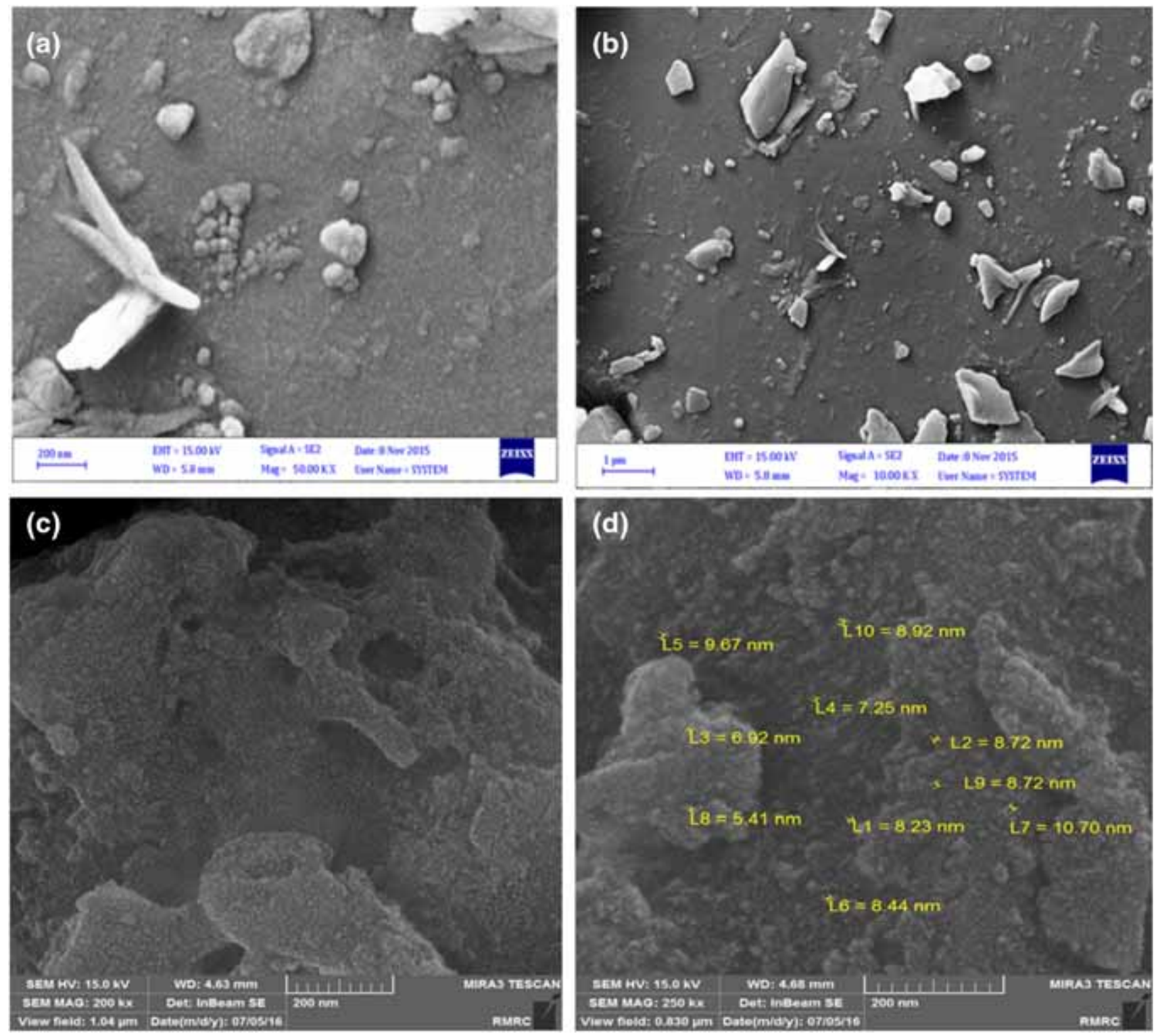

Figure 4. FE-SEM images of n-GW (a, b), and n-GW/PMA (c, d). 


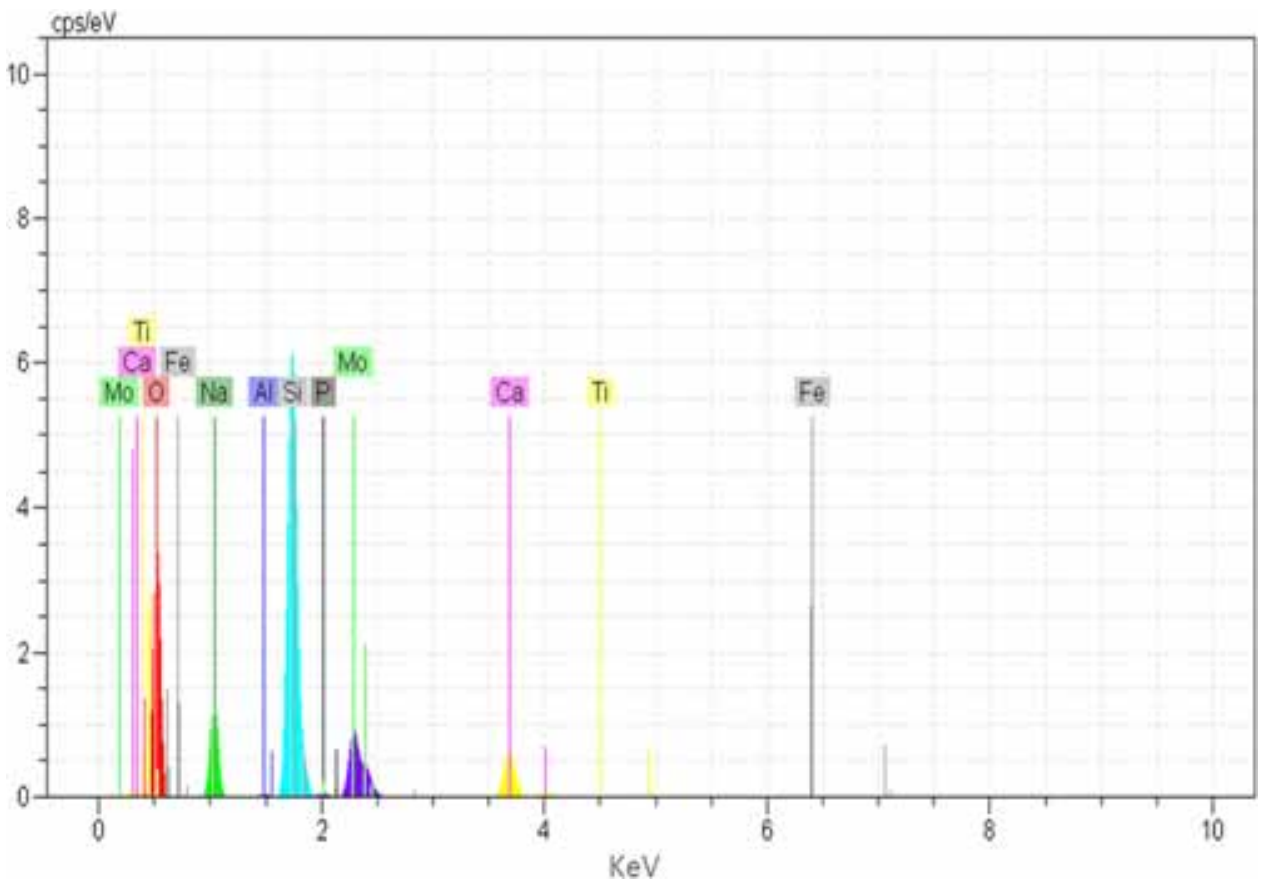

Figure 5. The EDX spectrum of n-GW/PMA 16 wt.\%.

Table 2. Comparison of n-GW/PMA for the oxidation of methyl phenyl sulfide with other reported procedures.

\begin{tabular}{lcccc}
\hline Entry & Reagent & $\mathrm{T}[\mathrm{min}]$ & Yield [\%] & Ref. \\
\hline 1 & Thiourea dioxide/TBHP & 210 & 93 & 39 \\
2 & $\mathrm{NaBO}_{3} .4 \mathrm{H}_{2} \mathrm{O} /$ Silica Sulfuric acid/ $\mathrm{KBr}$ & 840 & 55 & 40 \\
3 & Borax $/ \mathrm{H}_{2} \mathrm{O}_{2}$ & 360 & 35 & 41 \\
4 & Clay-supported ceric ammonium nitrate $(\mathrm{CAN}) / \mathrm{O}_{2}$ & 300 & 35 & 42 \\
5 & $\mathrm{SiO}_{2}-\mathrm{W}_{2}-\mathrm{Py} / \mathrm{H}_{2} \mathrm{O}_{2}$ & 150 & 90 & 43 \\
6 & $\mathrm{n}-\mathrm{GW} / \mathrm{PMA} / \mathrm{H}_{2} \mathrm{O}_{2}$ & 15 & 96 & Present work \\
\hline
\end{tabular}

safely employed in organic reactions at temperatures up to $350^{\circ} \mathrm{C}$.

\section{1d Field emission scanning electron microscopy:} (FE-SEM): FE-SEM images were employed in order to study the surface morphology, particle shape and size distribution of the as-synthesized materials (Figures $4 a-e)$. Figures $6 a$ and $b$ show the SEM images of the $\mathrm{n}-\mathrm{GW}$ which indicated that the material consisted of some particles on the surface. These nonhomogeneous particles have the sizes in the range of $50 \mathrm{~nm}$ to $2 \mu \mathrm{m}$. On the other hand, as displayed in the Figures $4 \mathrm{c}$ and d, n-GW/PMA had completely different morphology and size ranges. As it was obvious in the micrographs, after the modification of n-GW with PMA, the obtained material had aggregated particles with quasi-spherical shape. Besides, the size distribution of the catalyst particles was nearly homogeneous and their diameter sizes were around 5$11 \mathrm{~nm}$. 3.1e Energy-dispersive X-ray spectroscopy (EDX): The elemental composition of the n-GW/PMA from the EDX spectrum is depicted in the Figure 5. As it is obvious, the $\mathrm{O}, \mathrm{Si}, \mathrm{Al}, \mathrm{Ca}, \mathrm{Fe}, \mathrm{Ti}, \mathrm{Na}, \mathrm{P}$ and Mo elements ae present in the structure of the as-synthesized catalyst.

Table 2 displays the efficiency of our designed catalytic system in comparison with those reported in the literature. The result for the preparation of (methylsulfinyl benzene) in the presence of n-GW/PMA are compared with the data from the literature, which shows that the n-GW/PMA is superior to most of the others regarding the reaction times and product yields. Therefore, it could be concluded that n-GW/PMA is a very beneficial and efficient catalyst for the sulfide oxidation.

\subsection{Plausible mechanism for the oxidation of sulfides to sulfoxides catalyzed by $n-G W / P M A$}

Based on earlier reports, ${ }^{44,45}$ we propose a mechanism for the oxidation of sulfides to sulfoxides in the presence 


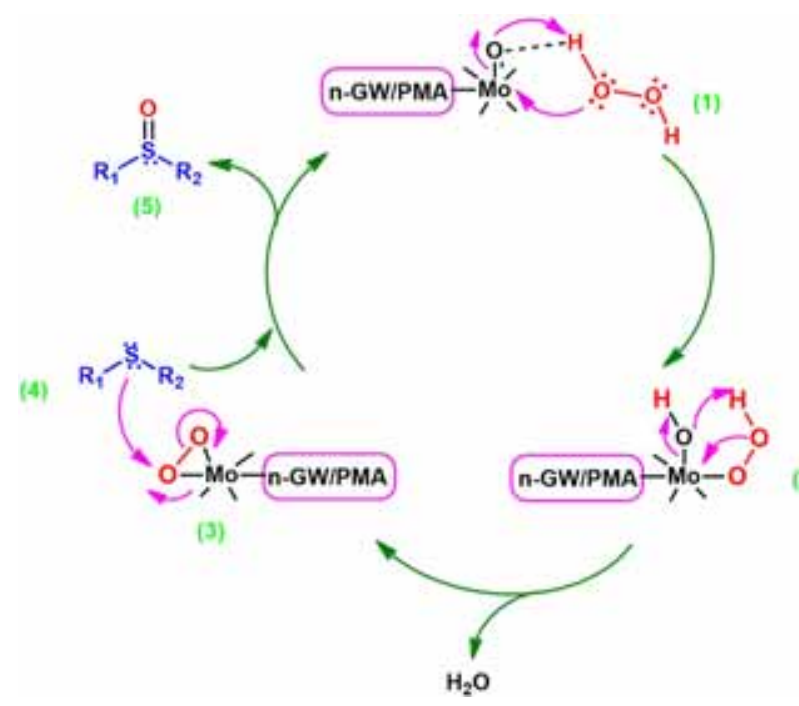

Scheme 3. Plausible mechanism for the oxidation of sulfides to sulfoxides in the presence of n-GW/PMA.

of n-GW/PMA (Scheme 3). The reaction is initiated with the nucleophilic attack of $\mathrm{H}_{2} \mathrm{O}_{2}$ on the oxo ligands of $\mathrm{Mo}(\mathrm{VI})$ atom on the $\mathrm{n}-\mathrm{GW}$ surface (i.e., species 1) to form the octahedral hydroperoxymolybdate (2). Then, monoperoxo species (3) is generated, followed by a loss of a water molecule. As a consequence, the peroxo group is electrophilically activated through the coordination to the high-valent molybdenum atom. Finally, the sulfoxide product (5) is formed when the sulfur atom in the organosulfur compounds (4) attacks on a peroxo group of intermediate (3) and regenerates polymolybdate or monoperoxo species.

\subsection{Reusability of the designed catalyst}

On account of the fact that the development of novel heterogeneous catalysts with the capability of good

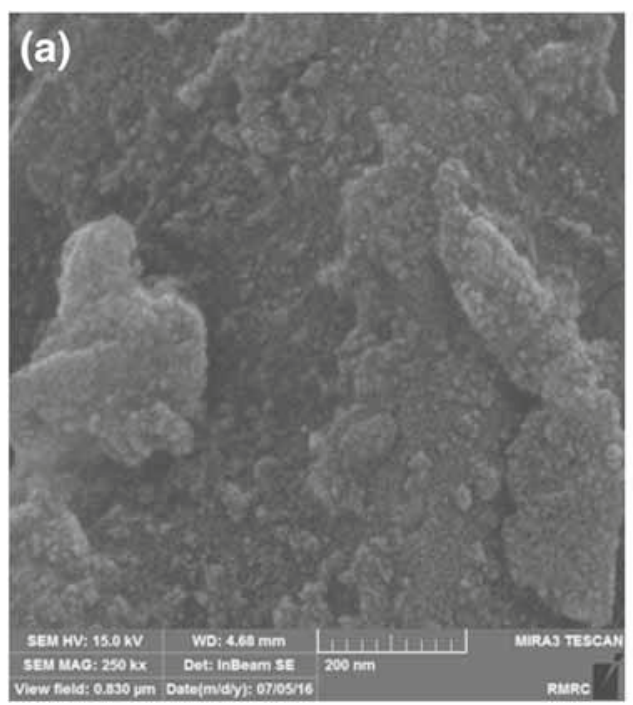

recyclability has been one of the important criteria for organic transformations, we examine our new nGW/PMA catalyst for its recyclability and reusability in the optimum reaction condition of selective sulfide oxidation. After each run, the catalyst was simply separated from the product by adding diethyl ether and, then by centrifugation. After that, the recovered catalyst was washed with extra amounts of ether and dried at $100^{\circ} \mathrm{C}$. The dried recovered catalyst was capable to efficiently run the oxidation reaction for five runs without losing its activity. The yields in the first 5 runs were as follows: 97 , 96, 94, 93 and $93 \%$. These results indicated the fact of tight immobilization of the PMA function on the $\mathrm{n}-\mathrm{GW}$ support.

The identity of the recovered catalyst was checked by FE-SEM which suggested that the nature of the catalyst remained intact and there was no change in the morphology during the reaction and recycling stages as compared to the fresh catalyst (Figure 6). Additionally,

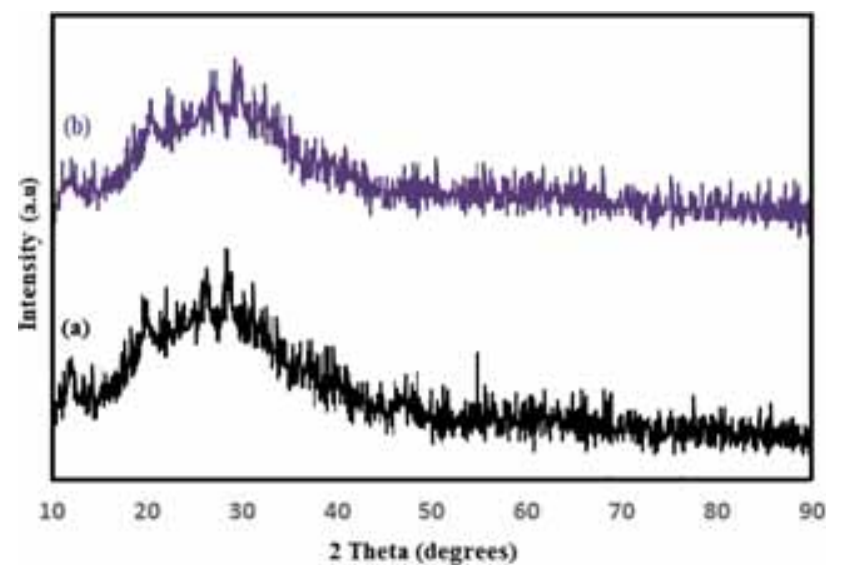

Figure 7. XRD spectra of n-GW/PMA (a) before use and (b) after reuse for five times.

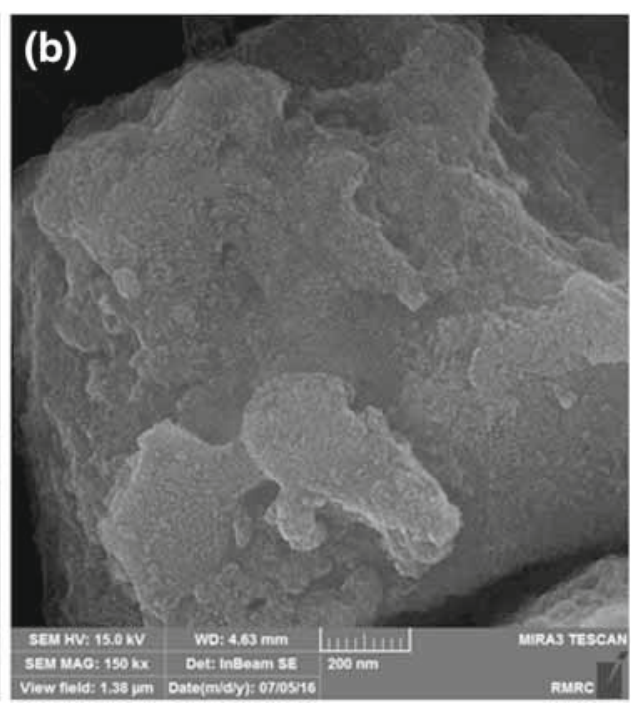

Figure 6. FE-SEM images of (a) fresh n-GW/PMA and (b) after $5^{\text {th }}$ run. 
the XRD patterns of the catalyst after five cycles were similar to the corresponding pattern before catalytic reaction which confirmed the existence of heteropolyacid groups on the surface of catalyst (Figure 7).

\section{Conclusions}

In conclusion, n-GW/PMA was synthesized as the eco-friendly, heterogeneous solid acid catalyst through the utilization of n-glass waste as an accessible and inexpensive solid support for the immobilization of phosphomolybdic acid. The strong binding of the PMA molecules on the surface of the n-GW/PMA caused the novel catalyst to show extraordinary activity and chemoselectivity for the synthesis of sulfoxides through the oxidation of sulfides. The designed reaction was green and economical since this transformation possessed several advantages, such as, short reaction times, high reaction yields, application of low amount of catalyst, solvent-free condition at room temperature, use of waste material as catalyst support, and utilization of $\mathrm{H}_{2} \mathrm{O}_{2}$ as the green oxidant.

\section{Supplementary Information (SI)}

All additional information pertaining to characterization of some of the products (Table 1) using IR spectra (Figures S1, S3, S7, S10 and S15), ${ }^{1} \mathrm{H}$ NMR spectra (Figures S2, S4, S8, $\mathrm{S} 11$ and S16) and ${ }^{13} \mathrm{C}$ MNR spectra (Figures S13 and S17), and details for the optimization of the reaction conditions by CCD method (Figures S18 and S19 and Tables S1, S2 and S3) are given in the Supporting Information, which is available at www.ias.ac.in/chemsci.

\section{Acknowledgements}

The authors would like to thank Semnan University Research Council for the financial support of this work.

\section{References}

1. Dioos B M L, Vankelecom I F J and Jacobs P A 2006 Aspects of immobilisation of catalysts on polymeric supports Adv. Synth. Catal. 3481413

2. Leadbeater N E and Marco M 2002 Preparation of polymer-supported ligands and metal complexes for use in catalysis Chem. Rev. 1023217

3. Rase H F 2000 Handbook of commercial catalysts: Heterogeneous catalysts (Boca Raton: CRC press)

4. Astruc D, Lu F and Aranzaes J R 2005 Nanoparticles as recyclable catalysts: the frontier between homogeneous and heterogeneous catalysis Angew. Chem. Int. Ed. 44 7852

5. Yoon T J, Lee W, Oh Y S and Lee J K 2003 Magnetic nanoparticles as a catalyst vehicle for simple and easy recycling New J. Chem. 27227
6. Zhu Y, Lee C N, Kemp R A, Hosmane N S and Maguire J A 2008 Latest developments in the catalytic application of nanoscaled neutral group 8-10 metals Chem. Asian J. 3650

7. Kozhevnikov I V 1998 Catalysis by heteropoly acids and multicomponent polyoxometalates in liquid-phase reactions Chem. Rev. 98171

8. Mizuno N and Misono M 1998 Heterogeneous catalysis Chem. Rev. 98199

9. Misono M 2001 Unique acid catalysis of heteropoly compounds (heteropolyoxometalates) in the solid state Chem. Commun. 1141

10. Palermo V, Villabrille P I, Vazquez P G, Caceres C V, Tundo P and Romanelli G P 2013 Role of vanadium and pyridine in heteropolycompounds for selective oxidation of alcohols with hydrogen peroxide J. Chem. Sci. 125 1375

11. Damyanova S, Fierro J L G, Sobrados I and Sanz J 1999 Surface behavior of supported 12-heteropoly acid as revealed by nuclear magnetic resonance, X-ray photoelectron spectroscopy, and Fourier transform infrared techniques Langmuir 15469

12. Kim H J, Chu Y H, Moon J H, Han H S and Shul Y G 2001 Preparation of heteropoly acid entraped in nano silica matrix Mol. Cryst. Liq. Cryst. 371131

13. Staiti P, Freni S and Hocevar S 1999 Synthesis and characterization of proton-conducting materials containing dodecatungstophosphoric and dodecatungstosilic acid supported on silica J. Power Sources 79250

14. Yan X M, Lei J H, Liu D, Wu Y C and Liu W 2007 Synthesis and catalytic properties of mesoporous phosphotungstic acid $/ \mathrm{SiO}_{2}$ in a self-generated acidic environment by evaporation-induced self-assembly Mater. Res. Bull. 421905

15. Dubey N, Rayalu S S, Labhsetwar N K and Devotta S 2008 Visible light active zeolite-based photocatalysts for hydrogen evolution from water Int. J. Hydrogen Energy 335958

16. Abdollahi-Alibeik $\mathrm{M}$, Zaghaghi $\mathrm{Z}$ and Mohammadpoor-Baltork I 2008 Alumina Supported 12Tungstophosphoric Acid as an Efficient and Reusable Catalyst for Synthesis of 1, 5-Benzodiazepines J. Chin. Chem. Soc. $\mathbf{5 5} 1$

17. Dupont P, Védrine J C, Paumard E, Hecquet G and Lefebvre F 1995 Heteropolyacids supported on activated carbon as catalysts for the esterification of acrylic acid by butanol Appl. Catal., A 129217

18. Engin A, Haluk H and Gurkan K 2003 Production of lactic acid esters catalyzed by heteropoly acid supported over ion-exchange resins Green Chem. 5460

19. Shayan A and Xu A 2004 Value-added utilisation of waste glass in concrete Cement Concrete Res. 34 81

20. Kolvari E, Zolfagharinia S, and Koukabi N 2016 A unique opportunity for the utilization of glass wastes as a resource for catalytic applications: toward a cleaner environment RSC Adv. 6113844

21. Hosseini MM, Kolvari E, Koukabi N, Ziyaei M and Zolfigol M A 2016 Zirconia Sulfuric Acid: An Efficient Heterogeneous Catalyst for the One-Pot Synthesis of 3,4Dihydropyrimidinones Under Solvent-Free Conditions Catal. Lett. 1461040 
22. Kolvari E, Koukabi N and Armandpour O 2014 A simple and efficient synthesis of 3,4-dihydropyrimidin-2-(1H)ones via Biginelli reaction catalyzed by nanomagneticsupported sulfonic acid Tetrahedron 701383

23. Kolvari E, Koukabi N and Hosseini M M 2015 Perlite: A cheap natural support for immobilization of sulfonic acid as a heterogeneous solid acid catalyst for the heterocyclic multicomponent reaction J. Mol. Catal. A: Chem. 39768

24. Kolvari E, Koukabi N, Hosseini M M and Khandani Z 2015 Perlite: an inexpensive natural support for heterogenization of $\mathrm{HBF}_{4}$ RSC Adv. 536828

25. Kolvari E, Koukabi N, Hosseini M M, Vahidian M and Ghobadi E 2016 Nano-ZrO2 sulfuric acid: a heterogeneous solid acid nano catalyst for Biginelli reaction under solvent free conditions RSC Adv. 67419

26. Kolvari E and Zolfagharinia S 2016 A waste to wealth approach through utilization of nano-ceramic tile waste as an accessible and inexpensive solid support to produce a heterogeneous solid acid nanocatalyst: to kill three birds with one stone RSC Adv. 693963

27. Koukabi N, Kolvari E, Khazaei A, Zolfigol M A, Shaghasemi BS, and Khavasi H R 2011 Hantzsch reaction on free nano- $\mathrm{Fe}_{2} \mathrm{O}_{3}$ catalyst: excellent reactivity combined with facile catalyst recovery and recyclability Chem. Commun. 479230

28. Koukabi N, Kolvari E, Zolfigol M A, Khazaei A, Shaghasemi B S, and Fasahatib B 2012 A Magnetic Particle-Supported Sulfonic Acid Catalyst: Tuning Catalytic Activity between Homogeneous and Heterogeneous Catalysis Adv. Synth. Catal. 3542001

29. Carreño M C 1995 Applications of sulfoxides to asymmetric synthesis of biologically active compounds Chem. Rev. 951717

30. Fernández de la Pradilla R, Colomer I and Viso A 2012 Sulfinyl-Mediated Stereoselective Overman Rearrangements and Diels-Alder Cycloadditions Org. Lett. 14 3068

31. An D, Guo Y, Zhu Y and Wang Z 2010 A green route to preparation of silica powders with rice husk ash and waste gas Chem. Eng. J. 162509

32. Wang B, Zhang J, Zou X, Dong H and Yao P 2015 Selective oxidation of styrene to 1, 2-epoxyethylbenzene by hydrogen peroxide over heterogeneous phosphomolybdic acid supported on ionic liquid modified MCM-41 Chem. Eng. J. 260172

33. Galvão A C P, Farias A C M and Mendes J U L 2015 Characterization of waste of soda-lime glass generated from lapping process to reuse as filler in composite materials as thermal insulation Cerâmica $\mathbf{6 1} 367$

34. Bhat A H, Khalil H P S A, Mishra R K, Datt M and Banthia A K 2011 Development and material properties of chitosan and phosphomolybdic acid-based composites $J$. Compos. Mater. 4539
35. Titus E, Ali N, Cabral G, Gracio J, Babu P R and Jackson M J 2006 Chemically functionalized carbon nanotubes and their characterization using thermogravimetric analysis, fourier transform infrared, and raman spectroscopy J. Mater. Eng. 15182

36. Rocchiccioli-Deltcheff C, Amirouche M and Fournier M 1992 Structure and catalytic properties of silicasupported polyoxomolybdates III. 12-molybdosilicic acid catalysts: vibrational study of the dispersion effect and nature of the Mo species in interaction with the silica support J. Catal. 138445

37. El-Wahab M M M A and Said A A 2005 Phosphomolybdic acid supported on silica gel and promoted with alkali metal ions as catalysts for the esterification of acetic acid by ethanol J. Mol. Catal A: Chem. 240109

38. Misono M, Mizuno N, Katamura K, Kasai A, Konishi Y, Sakata K, Okuhara T and Yoneda Y 1982 Catalysis by heteropoly compounds. III. The structure and properties of 12-heteropolyacids of molybdenum and tungsten $\left(\mathrm{H}_{3} \mathrm{PMo}_{12}\right)$ and their salts pertinent to heterogeneous catalysis Bull. Chem. Soc. Jpn. 55400

39. Kumar S, Verma S, Jain S L and Sain B 2011 Thiourea dioxide (TUD): a robust organocatalyst for oxidation of sulfides to sulfoxides with TBHP under mild reaction conditions Tetrahedron Lett. 523393

40. Habibi D, Zolfigol M A, Safaiee M, Shamsian A and Ghorbani-Choghamarani A 2009 Catalytic oxidation of sulfides to sulfoxides using sodium perborate and/or sodium percarbonate and silica sulfuric acid in the presence of KBr Catal. Commun. 101257

41. Hussain S, Bharadwaj S K, Pandey R and Chaudhuri M K 2009 Borax-Catalyzed and pH-Controlled Selective Oxidation of Organic Sulfides by $\mathrm{H}_{2} \mathrm{O}_{2}$ : An Environmentally Clean Protocol Eur. J. Org. Chem. 2009 3319

42. Dhakshinamoorthy A and Pitchumani K 2009 Claysupported ceric ammonium nitrate as an effective, viable catalyst in the oxidation of olefins, chalcones and sulfides by molecular oxygen Catal. Commun. 10872

43. Shi X Y and Wei J F 2008 Selective oxidation of sulfide catalyzed by peroxotungstate immobilized on ionic liquid-modified silica with aqueous hydrogen peroxide J. Mol. Catal. A: Chem. 280142

44. García-Gutiérrez J L, Fuentes G A, Hernández-Terán M E, Garcia P, Murrieta-Guevara F and Jiménez-Cruz F 2008 Ultra-deep oxidative desulfurization of diesel fuel by the $\mathrm{Mo} / \mathrm{Al}_{2} \mathrm{O}_{3}$ system: The effect of system parameters on catalytic activity Appl. Catal., A 334 366

45. García-Gutiérrez J L, Fuentes G A, Hernández-Terán M E, Murrieta F, Navarrete J and Jiménez-Cruz F 2006 Ultra-deep oxidative desulfurization of diesel fuel with $\mathrm{H}_{2} \mathrm{O}_{2}$ Appl. Catal., A 30515 